\title{
Some effects of light on sound intensity generalization'
}

DONALD D. DORFMAN AND RALPH MILLER

SAN DIEGO STATE COLLEGE

\begin{abstract}
Abstraet
This study determined whether a sound intensity generalization gradient would be displaced laterally if extraneous light intensity were changed from training to test. The results showed that (a) when Ss were trained in the absence of a light, introduction of the light on generalization-test trials displaced the generalization gradient toward the weaker sound intensities, and (b) when Ss were trained in the presence of a light, omission of the light on generalization-test trials displaced the gradient toward the larger sound intensities. Problem
\end{abstract}

Zajonc \& Dorfman (1964) and Zajonc \& Cross (1965) have reported a lateral displacement of the generalization gradient when extraneous stimulus intensity or drive level was changed from training to test. The major goal of this study is to further explore the effects of extraneous stimulus intensity. The only published study on extraneous stimuli and displacement of the gradient was one in which the generalization dimension was shock intensity. Since this particular dimension may have some unique properties associated with it, this study used sound intensity as the generalization dimension and light as the extraneous stimulus.

\section{Method}

The Ss were 48 girls enrolled in San Diego State College. Five Ss were discarded for failing to learn the discrimination. The sound intensities were generated by a Hewlett-Packard audio-signal generator and delivered to dynamic earphones (Permoflux, PDR-8). Each $S$ was tested individually in sound-proof room (IAC, Model 400-A). Stimuli were presented automatically and responses were recorded automatically. The center light (C-light) was a sheet of milk glass illuminated by two fluorescent lamps (G.E.4W daylight) with an ignition voltage of about 250 volts a.c. and had a luminance of $152 \mathrm{ft}-\mathrm{L}$.

The Ss were divided into two groups. Both groups were given a three-stimulus discrimination problem $\left(61 \mathrm{db}, 66 \mathrm{db}, 71 \mathrm{db}\right.$ re $.0002 \mathrm{dyne} / \mathrm{cm}^{2}$ ) in which they were reinforced for making one response to the middle sound intensity of $66 \mathrm{db}$ and a different response to the other two sound intensities. All sound intensities were $1000 \mathrm{cps}$. Group 2 was given the C-light on all training trials, and Group 1 was trained in the absence of the C-light. When the C-light was presented, it always came on and terminated simultaneously with the sound. The particular procedure was adapted from Brown, Clarke \& Stein (1958) and has been used in studying spatial generalization. Ss were told that they were going to participate in a horse-betting situation in which they were to guess whether a particular horse would win or lose on each race of a series of races. During training, the $S$ was instructed as to whether the horse won or lost the race by flashing a left or right light after the response. Above each light, a sign saying "win" or "lose" was placed. For half the Ss in each group, the left light signified "win" and the right light "lose," with the opposite being the case for the other half of the Ss. The Ss indicated their choice by pressing the left (right) key if they thought the horse would win the race and the right (left) key if they thought the horse would lose the race. If the 61 or 71 $\mathrm{db}$ tone was presented, the horse always lost the race, and if the $66 \mathrm{db}$ tone was given, the horse always won the race. There were 60 training trials, 30 trials of $66 \mathrm{db}$, and 15 trials each of 61 and $71 \mathrm{db}$. After training, they were given four blocks of generalization-test trials. On each generalization-test trial, one of seven sound intensities $(61,62,64,66,67,69,71 \mathrm{db})$ was presented in the presence or absense of the C-light. ${ }^{2}$ No feedback was given on the test trials. Each test block consisted of the random presentation of all the sound intensities under both light conditions (14 trials). There were also four additional training trials in each test block, one trial on 61 and $71 \mathrm{db}$, and two trials on $66 \mathrm{db}$.

The interval between onset of sound and onset of the reinforcing lamp was $3 \mathrm{sec}_{\circ}$, and the intertrial interval was $15 \mathrm{sec}$.

\section{Results and Diseussion}

Before considering the results on the generalization tests, it should be noted that Group 2 was more accurate than Group 1 during training as evaluated by analysis of variance, $F(1,46)=4.20, p<.05$. In fact, all of the non-learners were in Group 1. This result suggests that the C-light may have facilitated performance on the discrimination.

Figure 1 shows the post-discrimination generalization gradients under the no C-light and C-light conditions. As can be seen, in Group 1 (no C-light training) presentation of the $\mathrm{C}$-light shifted the gradient toward the lesser physical intensities, and in Group 2 (C-light training) omission of the C-light shifted the gradient toward the larger physical intensities. This lateral displacement with change in extraneous stimulation from training to test agrees with the results reported by Zajonc \& Dorfman (1964) on the generalization of shock intensity when the extraneous stimulus was 


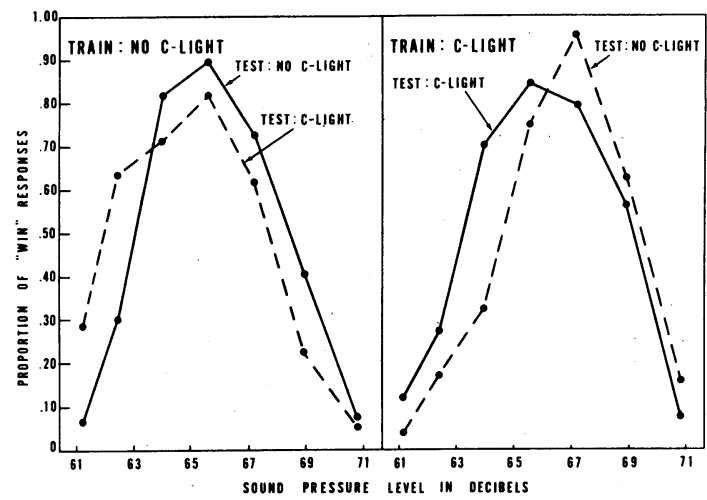

Fig. 1. Proportion of "win" responses as a function of sound pressure level. The solid lines were used to represent the test gradients obtained with the light condition the same in training and test.

sound. For statistical analysis of the generalization data, each S's distribution of responses to the sound intensities was treated as a frequency distribution and the mean of each distribution was computed for the no C-light and C-light gradient on each S. Displacement of the gradient was indicated by a difference in the mean of the gradients under the two conditions. In the analysis of variance, each $\mathrm{S}$ had two scores, a mean of the gradient of the no C-light condition and a mean of the C-light condition. Analysis of variance performed on these data revealed a significant Training effect, $F(1,41)=27.14, p<.001$ with the mean of Group 1 smaller than the mean of Group 2 (see Fig. 1). There was also a significant Test effect (test under no C-light vs. C-light), $F(1,41)=56.52, p<.001$. In both groups, the gradient under C-light test was displaced to the left of the gradient under the no C-light test. This significant Test effect provides statistical evidence for the observed lateral displacements. Analysis of variance of the modes of each gradient gave similar results. Ther vas a significant Training effect, $F(1,41)=19.45, p<.001$, and Test effect, $\mathrm{F}(1,41)=32.69, \mathrm{p}<.001$.

If the gradients are displaced in the manner described, the left wing of the gradient obtained on C-light test trials should have more "win" responses than the left wing of the gradient obtained on no C-light test trials in both groups. The reverse should be observed on the right wing. Analysis of variance of the arcsine transformation of the proportion of "win" responses on the left and right wing (excluding the middle stimulus of $66 \mathrm{db}$ ) confirmed this implication. Of particular interest was the reliable Test $X$ Wing effect, $F(1,41)=49.96, p<.001$. Simple comparisons showed that the mean proportion of "win" responses was higher under the C-light test on the left wing and higher under the no C-light test on the right wing. There was also a significant Training by Test by Wing interaction, $F(1,41)=9.87, p<.01$. The Test by Wing interaction was significant in both groups, but somewhat larger in Group 2. In conclusion, these data show that the finding of a lateral displacement of the gradient with introduction of extraneous stimuli is not restricted to generalization of shock intensity。

\section{References}

Brown, J. S., Clarke, F. R., \& Stein, L. A new technique for studying spatial generalization with voluntary responses. J. exp. Psychol., 1958, 55, 359-362.

Zajonc, R. B., \& Cross, D. V. Stimulus generalization as a function of drive shift. J. exp. Psychol., 1965, 69, 363-368.

Zajonc, R. B., \& Dorfman, D. D. Perception, drive, and behavior theory. Psychol. Rev., 1964, 71, 273-290.

\section{Notes}

1. This research was supported by a grant from the National Institute of Mental Health, MH 10449-01.

2. Unequally spaced stimuli should not affect the major results. 